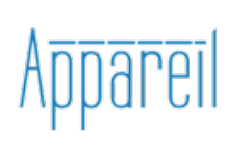

Appareil

$21 \mid 2019$

Le théâtre comme appareil

\title{
The Artist is Present : la théâtralité à l'origine de l'appareil perceptif
}

\section{Sunga Kim}

\section{(2) OpenEdition}

1 Journals

\section{Édition électronique}

URL : http://journals.openedition.org/appareil/3085

DOI : 10.4000/appareil.3085

ISSN : 2101-0714

Éditeur

MSH Paris Nord

Référence électronique

Sunga Kim, «The Artist is Present : la théâtralité à l'origine de l'appareil perceptif », Appareil [En ligne], 21 | 2019, mis en ligne le 23 mai 2019, consulté le 30 juillet 2020. URL : http://

journals.openedition.org/appareil/3085; DOI : https://doi.org/10.4000/appareil.3085

Ce document a été généré automatiquement le 30 juillet 2020.

\section{(c) (i) ()}

Appareil est mis à disposition selon les termes de la Licence Creative Commons Attribution - Pas d'Utilisation Commerciale - Pas de Modification 4.0 International. 


\title{
The Artist is Present : la théâtralité à l'origine de l'appareil perceptif
}

\author{
Sunga Kim
}

\section{Introduction}

1 Les médias visuels, de leur conception à leur réalisation, sont le fruit d'une réflexion sur notre façon de percevoir le monde. Il en résulte une imitation de ce que nous voyons - ou avons déjà vu - ou au contraire la création d'un outil d'élargissement de notre vision, nous permettant soit de mieux saisir le réel, soit de tenter d'appréhender une forme d'imaginaire. L'évolution de ces médias a de fait été fortement conditionnée par la nature du regard, au même titre qu'elle en a elle-même influencé les transformations au cours de l'histoire récente. Pour le dire de manière schématique, la plupart de ces médias traduisent un rapport plus ou moins équilibré entre deux approches, selon leur manière de traiter la perception de leur public et en dehors de toutes considérations commerciales : tantôt les créateurs se concentrent sur l'effet de projection proposé sans forcément prendre en compte la position des spectateurs, offrant ainsi une vision du contenu de ces médias plus ou moins contrainte et conventionnelle; tantôt ils se focalisent sur les divers enjeux de la réception, tentant souvent une démarche plus expérimentale pour que chacun approche l'œuvre à sa façon et en réalise sa propre vision.

2 Le théâtre quant à lui - de par sa nature artistique, sa configuration en vis-à-vis et les relations qui en découlent - est le lieu privilégié de la recherche d'un rapport particulier entre ces deux approches. Il a en effet, depuis sa fondation, sanctuarisé les réflexions autour du regard, chargeant la position du spectateur d'une importance primordiale ${ }^{1}$ et installant ainsi une relation de domination de celui qui voit sur celui qui fait $^{2}$. Aussi, et du fait du déroulement simultané de l'acte et de sa réception, cette position des spectateurs y est considérée comme une condition nécessaire qui complète la représentation, lui conférant un caractère unique et une certaine imprévisibilité. Cet acte du regard porté sur la scène est devenu emblématique et crée une tension telle 
entre la scène et la salle, qu'il intègre, par extension métonymique, l'ensemble des activités perceptives du spectateur en une expérience synthétique.

Cette conception essentielle du regard s'applique, en pratique, à tout acte spectatoriel, pour peu que celui-ci présente une forme de théâtralité. Si le théâtre est un média ou un genre, la théâtralité est plutôt un phénomène ou un concept qui permet la diversité des expressions et des interprétations de l'acte théâtral. La théâtralité est en effet souvent entendue comme une extension du champ théâtral, exportant hors du lieu "théâtre », certaines de ses conventions, telles que la construction dramatique, la configuration spatiale, la relation entre regardant et regardé ou la perception simultanée, et permettant ainsi une réalisation scénique plus libre. Mais plus qu'un simple emprunt aux modes du théâtre, la théâtralité exprime également une démarche transgressive. $\mathrm{Du}$ fait du dépassement du cadre classique strictement appliqué dans le théâtre, elle se traduit souvent par une mutation de cette forme originelle. Ce phénomène de renversement, inhérent à la notion de théâtralité, a parfois aussi pour effet de révéler paradoxalement l'essence du théâtre en tant que média relationnel et communicatif, lui permettant de se détacher des limites de la matérialité scénique.

4 Que ce soit par l'application d'éléments théâtraux ou la volonté de transgresser la forme théâtrale classique, la théâtralité aboutit entre autres à une diversification des conditions de perception de l'œuvre, tout en mettant l'accent sur la relation entre l'œuvre et le spectateur. C'est pourquoi nous explorerons, dans cet article, la théâtralité comme porteuse d'un nouveau point de vue perceptif, issu de son émancipation vis-à-vis de règles imposées par la condition théâtrale. Pour cela, il sera indispensable de faire un rapide point sur quelques théories de la théâtralité, afin de cerner la multitude de ses interprétations dans différentes disciplines. Alors seulement sera-t-il possible de s'interroger sur la possibilité, par le biais de la théâtralité, de parvenir à un état de modification de la perception de chaque spectateur. Dans ce cas, par quels moyens la théâtralité est-elle « injectée » dans l'œuvre, et à quoi aboutit-elle ? Toutes ces questions seront traitées à partir de l'exemple d'une performance de Marina Abramović : The Artist Is Present.

\section{La théâtralité, une notion complexe et transversale}

Le terme de " théâtralité » est souvent employé dans le langage courant pour exprimer la notion du théâtral, indiquant alors une situation particulièrement spectaculaire et dramatique, et une forme d'irréalité de ce qui est vu. Mais l'acception scientifique du terme, dont l'approche théorique est relativement récente ${ }^{3}$, a engendré des réflexions multiples qui lui ont chacune conféré leur définition propre. Du fait de ce développement épars, le concept de théâtralité reste aujourd'hui encore abstrait. De par son caractère hybride et sa nature transversale, il s'infiltre partout et se fait pluridisciplinaire, menant à une "dissolution des limites entre les genres et des distinctions formelles entre les pratiques ${ }^{4}$ », ce qui en fait une notion complexe, «floue et passe-partout ${ }^{5} »$.

Pluridisciplinaire et transversale, elle l'est sans aucun doute. Selon les minimalistes par exemple, et notamment Robert Morris ${ }^{6}$, la théâtralité réside dans la relation spatiale s'instaurant entre l'œuvre et le spectateur, et donne lieu à un dépassement du cadre classique et à la création d'œuvres aux dimensions monumentales. Ce gigantisme implique selon eux une participation physique : le spectateur doit choisir son point de 
vue sur l'œuvre - au sens premier du terme - en fonction de son positionnement et de ses déplacements. Michael Fried en a d'ailleurs fait une critique virulente dans son article « Art et objectité ${ }^{\text {» }}$, émettant l'idée que ces diverses perspectives contextuelles et cette théâtralité situationnelle étaient source d'une incomplétude de l'œuvre. Il y déplorait les effets d'une forme de théâtralité dans l'art plastique dont il concédait la réalité.

7 Pour les penseurs du théâtre, la théâtralité peut être vue comme une expression partielle du théâtre lui-même : elle en élargit paradoxalement le sens, en étendant les règles et en adaptant les conventions à diverses situations, comme l'évoque Patrice Pavis dans plusieurs de ses ouvrages ${ }^{8}$. Josette Féral, à l'inverse, définit la théâtralité par l'effet du regard qui fait de l'objet un signe, en s'appuyant sur l'idée d'une singularité du rapport entre l'objet et le sujet du regard ${ }^{9}$. Dans ses développements, elle montre aussi que l'autorité du seul regard du spectateur peut imposer la théâtralité à un objet sans aucune autre antériorité conditionnelle d'artificialité ou de contexte théâtral. Ce regard singulier instaure, selon l'auteure, une théâtralité due au "clivage spatial ${ }^{10}$ " entre le regardant et le regardé, menant à une altérisation des objets du regard, alors perçus comme appartenant à un "espace autre ${ }^{11}$ ". Roland Barthes résume la théâtralité comme suit : « c'est le théâtre moins le texte, c'est une épaisseur de signes et de sensations ${ }^{12}$ ", la définissant comme une " perception œcuménique ${ }^{13}$ » de tout ce qui est langage théâtral non textuel. Qu'elle soit conceptualisée par l'installation d'une œuvre ou imposée par l'effet du regard, la théâtralité révèle souvent la position du spectateur comme acteur de sa propre perception et actant de son regard, position qui va au-delà d'une simple identification/reconnaissance mais touche à une forme de participation à la complétion de ce qui sera l'œuvre.

$8 \mathrm{Au}$ vu de la diversité de sens véhiculée par le terme, l'idée de théâtralité pourrait donc être synthétisée selon deux axes : elle peut faire référence à la manière dont la scène existe dans l'acception d'une représentation plus extensive et libre, détachée de toute contrainte matérielle; ou développer l'idée d'un caractère théatral synthétique et multidisciplinaire, insistant notamment sur la relation entre l'œuvre et la position du spectateur et sur l'effet de leurs présences simultanées.

9 Partant de cette seconde idée d'une théâtralité liée à la participation par le regard, et afin d'en mieux saisir les qualités propres en tant qu'appareil perceptif conceptuel, nous étudierons dans les paragraphes suivants la performance The Artist is Present de Marina Abramović, réalisée en 2011 au MoMA (Museum of Modern Art) de New York ${ }^{14}$. Ce choix d'une performance - ni représentation théâtrale "artificielle», ni œuvre monumentale - pourrait sembler inapproprié pour illustrer certains effets de cette notion. On peut en effet penser qu'ajoutant en complexité, il risque de disperser le propos; mais cette œuvre est à ce point chargée, avec ses différents «niveaux de lecture ", de différentes expressions de la théâtralité, qu'elle constituera un excellent point de départ pour une réflexion sur l'influence de la théâtralité sur la perception des spectateurs.

10 D'une manière générale, la performance, bien qu'elle puisse être vue comme un genre dérivé du théâtre ${ }^{15}$, est par excellence un art du corps, de son expression physique et charnelle, faisant souvent de l'expérience de l'artiste l'unique sujet de réflexion. À l'inverse, The Artist is Present place le corps de l'artiste dans une posture particulière, suscitant un important mouvement participatif des spectateurs et un fort retentissement médiatique. Durant trois mois se tint une succession de rencontres 
entre l'artiste et ses spectateurs, défilant les uns après les autres, interrogeant l'acte du regard " concentré » et le rôle du corps exposé à ce regard. C'est de là que naîtra une des formes de théâtralité de cette œuvre, de cette capacité à engendrer une relation entre l'artiste et le spectateur, générant une expérience perceptive propre à chaque participant. C'est cela qui nous retiendra en premier lieu, ce jeu des regards, cette configuration en vis-à-vis invitant les spectateurs jusqu'au centre de l'événement performatif, de son mouvement. Ici, la théâtralité n'est pas elle-même sujet principal mais elle constitue une base indispensable, elle permet l'instauration des conditions nécessaires à la modification de la manière dont chaque spectateur approche et perçoit l'événement, établissant une relation intime et exceptionnelle entre l'œuvre et le spectateur.

\section{The Artist Is Present : une multitude de points de vue}

11 La performance The Artist Is Present, qui figurait au programme de la rétrospective éponyme consacrée à l'artiste, a eu lieu dans l'atrium du MoMA, salle grandement ouverte qui permet de nombreux accès et offre aux visiteurs une multitude de points de vue sur ce qui s'y passe. L'espace y est divisé en deux par une ligne blanche tracée au sol, formant un quadrilatère définissant immédiatement la zone de la performance, à l'intérieur, et la zone des visiteurs, invités à y prendre place librement. La zone intérieure est quasiment vide, mis à part les quelques éléments nécessaires au dispositif de la performance : une table ${ }^{16}$ et deux chaises installées en vis-à-vis au milieu de la salle, autour desquelles quatre projecteurs cinématographiques éclairent les rencontres. Ces dernières sont le contenu principal de cette performance et se déroulent de manière quasi simpliste : l'artiste reste assise à sa place, tête baissée, et ne lève son visage que pour regarder fixement le spectateur-participant qui vient s'asseoir devant elle, dans un défilé ininterrompu.

Dès l'organisation spatiale, aussi minimale que conceptuelle, la théâtralité s'impose comme un facteur primordial. La notion de «scène » est intensifiée par l'établissement $\mathrm{du}$ « lieu du regard», dans lequel tout ce qui arrive paraîtra «artificiel » du fait de la lumière blanche éblouissante affectant la qualité de la perception. Cette scénographie respecte plusieurs principes conventionnels du théâtre qui participent à l'instauration d'une ambiance dramatique renforcée par un effet de lumière qui charge l'objet à voir d'un dynamisme spectaculaire. Elle maintient, tout en les dérangeant, les deux positions des regardants et regardés, traditionnellement observées au théatre. Car l'agencement de ces deux fonctions permet ici de dépasser la condition du regard unilatéral (imposé par la forme classique) pour démultiplier la position des spectateurs libres de leurs mouvements.

13 L'espace carré central pousse en effet naturellement à un déplacement des visiteurs et suscite chez eux la recherche d'un point de vue idéal : derrière l'artiste, derrière le participant ou de manière à les voir tous les deux de profil, selon la perception qu'ils souhaitent avoir de l'œuvre. Une confrontation oblique et multiforme, due à une diversification du regard autant que de la perception, pourra ainsi être expérimentée par les visiteurs. Ceux qui le désirent pourront faire par eux-mêmes l'expérience des deux positions du vis-à-vis dont il est question dans cette rencontre: celle du participant et celle de l'artiste. 

haut concernant les cuvres monumentales minimalistes. En effet, cette relation particulière que critiquait Fried entre le spectateur et l'œuvre peut se retrouver dans la manière dont le spectateur-observateur perçoit la globalité de la performance. En choisissant ce qui selon lui constitue le « meilleur " point de vue pour saisir l'œuvre, il instaure, consciemment ou non, une relation particulière qui fait de son expérience spectatorielle un moment unique, quasiment non reproductible, et ne pouvant s'accomplir que par sa propre présence. C'est donc par sa présence et ses déplacements que l'œuvre pourra être complétée : en l'intégrant par son acte du regard, l'œuvre lui impose une participation tout en lui laissant la possibilité de l'aborder comme il le décide.

Mais cette liberté de mouvement accordée à tous les spectateurs agit encore à un autre niveau : lorsqu'un spectateur se déplace, il modifie non seulement sa propre perception de l'œuvre, mais aussi celle qu'en ont les autres, car les mouvements de son corps prennent place dans leur champ de vision. Aussi cette foule mouvante en arrière-plan de l'œuvre prend-elle les caractéristiques d'un groupe de spectateurs-figurants, ou comme le définit Georges Didi-Huberman d'une « toile de fond constituée de visages, de corps, de gestes ${ }^{17} »$. Chaque spectateur observateur reste non actant de l'œuvre bien que la collectivité visible et mobile qu'il forme avec les autres constitue un « décor humain » agissant sur la perception de tous. Ce mouvement d'autres corps perçus par le spectateur est d'autant plus visible qu'il a lieu dans un espace global respectant les conventions de ce que Brian O'Doherty nomme le white cube, à savoir un espace blanc, détachant l'œuvre de tout contexte extérieur et lui conférant un aspect sacré ${ }^{18}$. Là, chaque corps conditionne l'expérience spectatorielle en définissant différents centres d'attraction, d'une part car l'attention que chacun porte sur tel ou tel détail attire la curiosité des autres, de l'autre parce que les déplacements des premiers sont influencés par le positionnement et le mouvement des seconds. C'est ainsi avec un paysage spectatoriel vivant que les spectateurs-observateurs doivent composer afin de réaliser leur perception individuelle ${ }^{19}$.

16 C'est donc bien cette configuration spatiale, tant dans sa conception que dans sa réalisation, qui contribue à définir les relations entre le spectateur-observateur et l'œuvre, à la fois en lui imposant les éléments essentiels d'une perception propre et intime, mais aussi en lui permettant une liberté de mouvements et donc de points de vue. Cet espace permet également une mise en abîme de la théâtralité, car la figure des spectateurs est intégrée au champ de vision de tout un chacun, et que ce sont eux qui sont porteurs du dynamisme habituellement réservé à la scène. Et c'est finalement la « théâtralisation » de ce lieu, au sens où l'entendait Gilles Girard, Réal Ouellet et Claude Rigault $^{20}$, qui permet de tisser une relation scène-salle particulière et donc de créer une perception singulière de l'évènement par les spectateurs-observateurs.

\section{Le regard de l'artiste comme appareil primitif}

17 Cette participation, indirecte et périphérique, induisant une diversification de la perception de l'observateur, n'est de toute évidence pas la seule forme de participation présentée aux visiteurs. Une autre, plus directe et concrète, leur sera proposée dès le lancement de la performance, via les confrontations successives avec l'artiste qui sont le cœur de l'œuvre. Celles-ci exigeront un bouleversement quasi immédiat de leur 
position habituelle, celle de regardant. Les spectateurs s'intégreront cette fois dans le champ de la performance: chacun pourra vivre sa propre expérience de l'acte performatif et devenir une partie de l'œuvre.

Toute la performance est focalisée sur l'acte du regard, et tout doit s'y exprimer pendant la durée de chaque rencontre. Certaines règles de base sont strictement observées, autant par l'artiste que par le participant - aucun autre geste que le regard, seul moyen d'expression, n'est autorisé -, ce qui a pour effet de maintenir une ambiance silencieuse et quasi solennelle, tout à fait contrôlée. Une tension dramatique, un suspens presque, émergera du principal mouvement réellement apparent dans cette salle plongée dans une concentration disciplinée : celui, régulier et répété, de l'entrée et de la sortie des participants. L'artiste, quant à elle, y est en permanence présente comme l'indique le titre de l'œuvre - enchaînant sans interruption les rencontres, tandis que les participants lui font face volontairement et partent quand ils le souhaitent. Les conditions légèrement différentes qui sont imposées à ces deux présences (le participant subissant en effet moins de contraintes que l'artiste) renvoient l'image de deux positions interagissantes: le corps de l'artiste est exposé, soumis au regard des participants pouvant l'observer à loisir, comme s'il était objet inerte. Mais cette impression sera rapidement dissipée, et même renversée quand les participants, une fois installés, se confronteront réellement à l'artiste, renversement qui se renforce avec la durée du face-à-face.

19 En effet, en entrant d'eux-mêmes dans la zone de performance, en s'y intégrant, les participants changent la qualité de leur présence et adoptent une position analogue à celle de la performeuse. Dans les faits, tous leurs actes, leur présence même, deviennent « objets » mis à disposition du regard des autres, et se chargent du poids et du sens de la conception artistique. Mais le fait qu'un spectateur soit intégré ou s'intègre de luimême à une œuvre n'est en soi pas nouveau; le réel renversement proviendra du comportement singulier de l'artiste elle-même, dont la démarche est ici contraire à celle habituellement attendue de l'artiste comme corps exprimant.

Certes, malgré l'intégration volontaire du participant au champ performatif, celui-ci conserve partiellement son identité de regardant - de fait superposée à celle nouvellement acquise de regardé - et garde, en raison de l'immobilité de l'artiste, une part de son pouvoir spectatoriel. Il est alors une entité "invitée» de manière éphémère, dont le rôle consiste, au départ, principalement à "regarder » l'artiste présente face à lui, et ce de façon anonyme, c'est-à-dire sans qu'aucun statut spécifique ne lui soit proposé pour cette performance. Dans ce contexte, sa participation n'a rien à voir avec un acte conceptuel de "jeu» représentationnel, issu d'une technique d'incarnation d'un personnage détaché de soi, ou des phénomènes liés au travail de l'acteur.

21 Mais la neutralité du regard de l'artiste, constamment fixé sur chaque participant sans se détourner une seule seconde, remet en cause la fonction de regardant en le privant d'« acte à voir ", et estompe ainsi progressivement l'identité de regardant jusque-là partiellement conservée par le participant. La contrainte que l'artiste s'est imposée à elle-même peut, en effet, être comparée à celle de la figure symbolique des spectateurs dans le théâtre, du fait de son regard passif fixé sur ce qui remplace ici l'image scénique, celle-ci restant assise, immobile et silencieuse comme le récepteur d'une communication unilatérale. Elle se met ainsi en position de témoin qui capture l'image 
des participants, prenant de la distance par rapport à leurs «actes " manifestés et exprimés, si infimes soient-ils.

Aussi le participant est-il ici la seule entité « active » et spectaculaire, en opposition à l'artiste qui par son comportement «suspend " l'action. Contraignant le participant à extérioriser ses émotions par des expressions corporelles élémentaires, l'artiste lui restitue la charge de la communication de cette rencontre, afin qu'il s'impose une certaine pression expressive révélant constamment sa position de regardé. Sans aucune réponse de la part de l'artiste, le participant doit assumer ce temps qu'il s'assigne à luimême: soit il quitte cette position relativement inconfortable et inhabituelle, soit il profite des regards concentrés sur lui, s'amusant de cette situation dans laquelle il est la seule figure actante.

Les participants sont généralement plutôt réactifs et expressifs, chacun cherchant un moyen de communiquer dans ce contexte restreint, transmettant souvent leurs émotions sans hésitation, quelle qu'en soit la forme. Cette situation particulière les pousse à endosser leur rôle, à "jouer " chacun à leur manière. Beaucoup expriment leur ressenti par leurs expressions faciales et à travers leurs regards, projetant une part d'intimité, au point que soient identifiables, selon les cas : la jouissance, l'apaisement, le mécontentement, le doute etc. Beaucoup ne peuvent se retenir de verser des larmes, allant parfois jusqu'à attendre des heures durant de pouvoir prendre place ou à revenir plusieurs fois pour s'asseoir face à l'artiste et donc revivre cette expérience. Des comportements tout à fait inverses à ceux évoqués plus haut ont également pu être observés : certains participants ont essayé de s'affranchir des règles imposées par la performance, tentant ostensiblement de troubler l'artiste, comme s'ils voulaient que leur passage devienne un événement en lui-même, pour susciter l'intérêt ou faire parler d'eux ${ }^{21}$. L'« inexpression» de l'artiste fit ainsi étrangement surgir des réactions plus volontaires et concrètes chez les participants, leur donnant en quelque sorte le courage de l'expression ${ }^{22}$.

D'autre part, une fois passée la vague émotionnelle, l'occasion offerte aux participants de se concentrer sur leur seul ressenti pouvait les mener paradoxalement à quelque chose se rapprochant d'un état méditatif. Le regard de l'artiste fonctionne, après un certain temps passé à s'y plonger, comme un miroir leur renvoyant leur propre image. Marina Abramović expliquait dans un entretien à ce propos que les réactions des participants étaient le fait de cette projection de leur propre image qu'elle leur renvoyait ${ }^{23}$, et qu'elles n'avaient rien à voir avec sa présence. La réelle scène dramatique et théatrale se déploie ainsi au moment du plongeon dans la perception intime et de la retransmission de ces émotions partagées. Il s'agit ici d'une expérience perceptive déplacée par un glissement de l'état du spectateur, placé à la fois comme objet et sujet du regard, détournant son regard sur lui-même jusqu'à son moi intérieur et se focalisant sur ce moment particulier.

Le regard de l'artiste agit ici, en définitive, comme un appareil primitif qui permettra d'une part au participant de se percevoir autrement, et d'autre part au public assistant à la performance de percevoir, à travers le regard fixe de l'artiste, ce participant comme autre chose qu'un «simple spectateur ». Le regard de l'artiste est donc bien, dans ce contexte précis, un intermédiaire qui modifie la perception que chacun a de la réalité. L'artiste déclenche, par son regard, un autre regard de la part du participant, le révélant dans une réalité intérieure contextualisée chaque fois différemment, suspendu dans le temps et l'espace, le transportant momentanément vers un « ailleurs » intime. 
26 Le trouble des fonctions est donc un facteur indispensable pour qu'opère ce renversement des positions, qui suscite un changement du mécanisme de perception habituel menant à un autre état et à un autre degré du voir. Le regard traité comme sujet, dans cette performance jouant avec les règles et conventions théâtrales, semble pouvoir repousser les limites de son acte et permettre une vision «autre», plus intérieure. C'est ainsi que se trouve atteint un deuxième niveau de théâtralité, se développant de manière plus complexe et transgressive, imposée par une condition de rupture d'une situation "prévisible", mais aussi par la révélation d'une forme de perception méditative dépassant la projection concrète de la matérialité scénique. Le phénomène de retournement est ici installé par l'utilisation volontaire des codes théâtraux, finalement employés comme déclencheur d'une prise de conscience de l'essentialité de l'acte du regard.

Ce phénomène de déplacement de l'objet visuel principal, issu de l'« effacement » de la présence de l'artiste - sujet pourtant mis en avant dans le concept de départ de la performance - se poursuivra jusque dans la diffusion en ligne du portrait des participants, ie dans une ouverture du musée vers l'espace numérique, où l'absence de l'artiste ne sera plus seulement conceptuelle mais bel et bien concrète.

\section{Remédiation : une théâtralité nouvelle pour une remise en scène des émotions}

Il y a en effet un autre élément significatif, en regard de la relation entre le spectateur et l'œuvre, qui n'a pas encore été évoqué ici : la présence et le travail du photographe Marco Anneli. Il s'agit d'une collaboration photographique conçue avec Marina Abramović en amont de la performance, menant à une forme nouvelle d'expérience perceptive destinée aux spectateurs en dehors du musée, à travers une remise en scène de l'œuvre dans l'espace numérique. Le projet de Marco Anneli est une démarche parallèle dépassant le simple enregistrement photographique documentaire. Le photographe reproduit la confrontation des regards, question essentielle de la performance, dans un autre contexte médiatique. Par l'application d'une méthode rigoureuse, la performance sera reproduite et augmentée pour être vue et partagée par un public plus nombreux, offrant une expérience du regard diversifiée. Ce qui est particulièrement captivant ici, c'est la création par cette démarche d'une relation exclusivement inter-spectatorielle, dans laquelle la figure des spectateurs est l'unique présence et occupe toutes les positions, à la fois objet et sujet de l'expérimentation du regard.

De nombreuses images des spectateurs-participants ont en effet été méthodiquement et quotidiennement publiées sur le site du MoMA : celles captées par deux caméras vidéo filmant sans discontinuer l'ensemble de la scène de la performance pour une diffusion en direct, et celles enregistrées par l'appareil photo manipulé par M. Anneli réalisant les portraits de tous les participants ${ }^{24}$. Contrairement aux caméras fixes disposées latéralement pour une captation globale automatique, chaque portrait de participant est pris selon un angle réfléchi et calculé par le photographe, positionné derrière l'artiste pendant toute la durée de la performance ( 716 heures et 30 minutes au total), résistant à la douleur physique et à l'épreuve du temps au même titre que l'artiste. Les 1545 portraits réalisés ont été mis en ligne tous les soirs sur le site du MoMA par le biais de sa page Flickr ${ }^{25}$. Chaque portrait y défile par ordre chronologique, accompagné 
d'une note d'information précisant le jour, la durée et la date de participation. Cette somme d'images a donné lieu à un autre événement, extérieur au musée mais presque simultané, étroitement relié au temps et à l'espace performatif, tant physiquement que psychologiquement. Les images des différentes participations ont très rapidement été diffusées par les internautes qui les téléchargeaient, partageaient et republiaient, démultipliant ainsi leur audience alors même que les photographies personnelles sont interdites à tous les visiteurs présents dans la salle. Ce phénomène de renouvellement virtuel de la scène créera également un fort impact médiatique et renforcera l'intérêt du public pour l'événement dans son ensemble.

Techniquement, lorsque le photographe réalise ces portraits, il adopte un point de vue le plus proche possible de celui de Marina Abramović, cherchant à faire coïncider son objectif avec la position de l'œil de l'artiste. Avec le même soin que celui avec lequel il cherche la position idéale, il vise chaque fois la capture la plus détaillée qu'il puisse faire des expressions du participant, utilisant les éléments à sa disposition à des fins réalistes: la lumière intense projetée des quatre coins de l'espace augmente la précision des expressions faciales tout en effaçant toutes les ombres portées sur le visage ; le puissant téléobjectif monté sur son appareil donne une qualité d'image en très haute définition ${ }^{26}$.

31 Cette mise en image du participant fera l'effet d'une «remise en scène » numérique permettant aux internautes d'expérimenter le face-à-face au cœur de cette œuvre. La particularité de l'individualisme habituel des outils informatiques - un écran par utilisateur - est ici employée fort à propos, pour permettre une confrontation personnelle libérée de toute forme de communication ${ }^{27}$. La proximité ainsi obtenue sera similaire à celle vécue sur les lieux de la performance, sans que ne soit requise la participation effective de l'internaute, tout en renversant complètement les positions telles qu'observées au musée. En effet, comme la nouvelle position proposée aux internautes est finalement calquée sur celle de l'artiste et non du participant, ils " prennent sa place » et adoptent un point de vue auquel ils n'auraient jamais pu avoir accès lors du déroulement réel de la performance, se confrontant à un autre spectateur. L'artiste joue alors malgré elle à nouveau un rôle d'intermédiaire, mais sous une autre forme, aboutissant à sa disparition physique. In fine, seul le regard du spectateurinternaute fixe réellement celui du spectateur-participant, dans une tentative de transmission des "yeux " de l'artiste afin qu'étape après étape, une configuration de vis-à-vis entre celui qui regarde et celui qui exprime puisse avoir lieu : à travers l'œil de l'artiste, puis l'objectif de l'appareil photo dirigé par l'œil du photographe, le regard du spectateur-participant est transmis au spectateur-internaute, qui, ignorant tous ces intermédiaires invisibles pour lui, s'y trouve donc directement confronté.

32 Si l'on considère ici l'œil de l'artiste comme un «média » symbolique originel qui sera réinterprété par la démarche photographique, les portraits résultants de cette tentative de reproduction peuvent être vus comme une "remédiation ", c'est-à-dire comme l'expression d'un même contenu à travers un nouveau média. Ce phénomène de remédiation a souvent pour but, comme l'expliquent Jay David Bolter et Richard Grusin $^{28}$, d'offrir au récepteur une amélioration de la manière dont il accède à l'information, soit parce que le nouveau média est plus efficace, soit parce qu'il se rapproche de la réalité et devient plus "oubliable», au profit de l'information ellemême ${ }^{29}$. Mais cette hypothèse contient un paradoxe, car ce qui est considéré comme « média » originel, soit l'œil de l'artiste, ne pourra jamais être remplacé par un média 
plus performant, produisant un « meilleur » résultat, plus réaliste et pertinent que l'œil humain. Pourtant, ce processus de reproduction du point de vue de l'artiste permet au spectateur-internaute d'avoir accès à une part de réalité autrement inaccessible, et le principe de remédiation paraît donc pouvoir être appliqué à la démarche du photographe. En effet, en prenant soin de capturer, selon ses propos, «le moment où les participants versent leurs premières larmes ", et en choisissant attentivement les images les plus expressives pour les publier ${ }^{30}$, ce n'est pas la position de l'artiste ni la renommée de l'événement qu'il cherche à transmettre. C'est plutôt une révélation émotionnelle qu'il souhaite atteindre par ces regards, en déclenchant une réitération de l'« état " provoqué par les expériences de chaque participant lors de la rencontre initiale, basée sur une communication intime et individuelle. C'est ainsi à cet état émotionnel qu'ont accès les internautes plutôt qu'au «simple» point de vue de l'artiste, et c'est donc cela qui fait réellement ici l'objet d'une remédiation.

Cette transmission des émotions instaure, par le biais d'une prolongation de l'espace performatif dans le virtuel, une relation nouvelle entre le récepteur final de cette démarche et l'image du participant devenu simple objet du regard. Confronté à une succession d'émotions vives, décontextualisées et dont le nombre accentue le caractère exagéré, l'internaute devient à son tour la seule présence actante dans cette "remise en scène » de la confrontation de départ. Or, ce processus créateur d'émotions, dénué de texte ou de parole, apparaît comme une forme primitive du pathos, que Gilles Declercq décrit comme un " processus rhétorique par lequel l'orateur suscite l'émotion de l'auditoire ${ }^{31} »$. Ici, c'est donc par le seul jeu des regards que sera permise cette "mobilisation des passions de l'âme chez le spectateur ${ }^{32}$ ", les préparant à une possible expérience cathartique. Ainsi, une forme de théâtralité singulière est ici à l'œuvre pour les internautes. S'il était dans les paragraphes précédents question d'abord d'une théâtralité spatiale imposant des rôles prédéfinis, puis d'une théâtralisation de la présence du spectateur à travers le regard altérisant de l'artiste, c'est ici plutôt la transmission des émotions qui établit les conditions propices à l'émergence de la théâtralité.

\section{Conclusion}

Ces bouleversements à différents niveaux des positions attendues dans le déroulement de la performance ont donc été rendus possibles principalement du fait de deux facteurs: l'injection, délibérée ou non, de différentes formes de théâtralité dans l'œuvre et le glissement du point central d'intérêt de l'artiste vers le spectateur. En effet, Marina Abramović, a ici fait le choix de « rejouer » un défi artistique impliquant à l'origine deux performeurs, mais en modifiant une donnée primordiale: la place occupée originellement par son collaborateur est laissée libre pour être remplie par l'intégration volontaire d'un spectateur. Ce qui était au départ une confrontation permanente devient une performance participative fondée sur la rencontre. Ainsi, avant même que les participants n'entrent en jeu, plusieurs points font de cette expérience une œuvre particulière, à la fois corporelle, participative et synthétique de la rétrospective ${ }^{33}$ dans laquelle elle s'inscrit.

35 Le participant à cette pseudo-scène devient donc rapidement le centre d'intérêt, prenant la place du « principal protagoniste », occupée par Marina Abramović au début de la performance. Il devient celui qui est vu, celui dont on parle et qui obtient son 
« quart d'heure de célébrité ». Cette mise en avant de la participation du spectateur a été stratégiquement réfléchie lors du lancement de ce projet, et renforcée en plusieurs points par les effets d'une théâtralité sous-jacente et hétérogène. Qu'il s'agisse de celle issue de la relation du spectateur avec l'œuvre dans sa globalité, de celle naissant de la tension méditative entre le participant et l'artiste, ou de celle - indirecte mais indéniable - produite par la remise en scène des émotions concentrées dans la salle du musée transmises à une nouvelle figure du spectateur numérique, une théâtralité protéiforme joue sur la manière dont le récepteur de l'œuvre la perçoit, l'interprète et en fait une expérience intime et singulière. Toutes ces formes spectatorielles aboutissent, par l'implication plus ou moins prononcée qu'elles imposent, à une prise de conscience par le spectateur de sa position, de son rôle et même de son image qui aura inévitablement une influence sur son état de spectateur et sa perception de l'œuvre.

Mais si la théâtralité est un élément nécessaire aux conclusions obtenues dans ce cas précis, on pourrait encore se demander si elle n'est pas également une condition suffisante à la définition de l'œuvre artistique telle qu'elle est pratiquée aujourd'hui : à la fois transdisciplinaire et ouverte; participative et immersive, n'excluant plus le spectateur dans une position définie; et enfin connectée, ne pouvant pas ignorer les nouveaux outils de communication et leurs possibilités relationnelles quasi illimitées.

\section{BIBLIOGRAPHIE}

Akers Matthew et Dupre Jeff, Marina Abramović, The Artist Is Present, New York, HBO Documentary Films, 2012, DVD.

Banu Georges, Le Rouge et Or, une poétique du théâtre à l'italienne, Paris, Flammarion, 1989.

Barthes Roland, Essais critiques, Paris, Le Seuil, 1964.

Bénichou Anne, « Marina Abramović : The Artist Is [Tele] Present : les nouveaux horizons photographiques de la (re)performance ", Intermédiarités, Québec, $\mathrm{n}^{\circ}$ 17, 2011, p. 147-167. [En ligne] : https://doi.org/10.7202/1005754ar

Biet Christian et Christophe Triau, «La mise en scène : un héritage, des interrogations ", Qu'est-ce que le théâtre?, Paris, Gallimard, 2006.

Ciacchè Piergiorgio, «L'art du spectateur. Voir les sons et écouter les visions », in « Regard de l'autre, regard sur l'autre », Diogène, Paris, Puf, n 193, 2001, p. 100-113. [En ligne] : https:// www.cairn.info/revue-diogene-2001-1-page-100.htm

David Bolter Jayet et Grusin Richard, « Immediacy, Hypermediacy, and Remediation », Remediation: Understanding new media, Cambridge, MIT Press, 2000.

Davvetas Démosthènes, Marina Abramović : méditation aux yeux fixes, Paris, Éditions Nicolas Chaudun, coll. « Noème $12 », 2013$.

Declercq Gilles, « Pathos et théâtralité. Pour une économie cognitive des passions », in Rinn Michael (dir.), Émotions et discours : L'usage des passions dans la langue, Rennes, Presses 
universitaires de Rennes, 2008, p. 219-245, 2008 (généré le 20 février 2019). Disponible sur Internet : <http://books.openedition.org/pur/30443>. ISBN : 9782753546752. DOI : 10.4000/ books.pur.30443

Deleuze Gilles, « Chapitre 6, l'image-affection : visage et gros plan », Cinéma1 : L'image-mouvement, Paris, Les Éditions de Minuit, 1983.

Didi-Huberman Georges, Peuples exposés, peuples figurants. L'œil de l'histoire, 4, Paris, Les Éditions de Minuit, 2012.

Dumouchel Réjean, «Le spectateur et le contactile », in « Nouvelles technologies : nouveaux cinémas ?», Québec, Cinémas, vol. 1, n 3, 1991, p. 38-60. [En ligne] : https://www.erudit.org/fr/ revues/cine/1991-v1-n3-cine1501487/1001065ar/

Ekman Paul, Emotions Revealed: Understanding Faces and Feelings, New York, Times Books, 2003.

Féral Josette, « La théâtralité : la spécificité du langage théâtral », Poétique, n 75, Paris, Le Seuil, 1988, p. 347-361.

Féral Josette (dir.), Pratiques performatives. Body Remix, Québec/Rennes, PUQ/PUR, 2012.

Fernandez Laure, "Théâtralité et arts visuels : le paradoxe du spectateur. Autour de "The World as a Stage" et "Un teatre sense teatre" ", Marges [En ligne], 10| 2010, mis en ligne le 15 avril 2010. URL : http://journals.openedition.org/marges/490 ; DOI : 10.4000/marges.490 [consulté le 18/03/2019].

Fried Michael, « Art et objectité », in Contre la théâtralité. Du minimalisme à la photographie contemporaine, Paris, Gallimard, coll. « NRF essais », 2007, traduit de l'anglais Art and Objecthood: essays and reviews par Fabienne Durand-Bogaert. .

Girard Gilles, Ouellet Réal et Claude Rigault, L’Univers du Théâtre, Paris, Puf, 1978.

Milgram Stanley, Soumission à l'autorité [Calmann-Lévy, 1994], Paris, Fayard/Pluriel, 2017.

Morris Robert, « Notes on Sculpture », Minimal Art, A Critical Anthology, Berkeley/Los Angeles/ Londres, University of California Press, 1995.

O’Doherty Brian, White Cube. L'espace de la galerie et son idéologie, Zurich, JRP Ringier, 2008.

Pavis Patrice, "Théâtralité en Avignon ", in Vers une théorie de la pratique théâtrale : voix et images de la scène, Paris, Presses Universitaires du Septentrion, 2000.

Pavis Patrice, Dictionnaire du théâtre, Paris, Armand Colin, 2004.

Popper Frank, «La créativité aujourd'hui », in Art, action et participation : l'artiste et la créativité [1980], Paris, Klincksieck, 2007.

Rey Alain (dir.), Dictionnaire historique de la langue française [1993], Paris, Le Robert (Édition numérique), 2011.

Salamé Nadine, L'Hyperréalité du monde postmoderne selon Jean Baudrillard, Paris, L'Harmattan, 2016.

\section{NOTES}

1. D'après le Dictionnaire historique de la langue française, le mot théâtre « est emprunté (mil. $\mathrm{XII}^{\mathrm{e}} \mathrm{s}$.) au latin classique theatrum "lieu de représentation" [...], lui-même emprunté au grec theatron. Ce mot est dérivé de thea "action de regarder", "vue, spectacle, contemplation", que l'on rapproche à l'intérieur de la langue grecque de thauma "merveille" (thaumaturge).». Alain Rey (dir.), 
"Théâtre », Dictionnaire historique de la langue française [1993], Paris, Le Robert (Édition numérique), 2011, p. 9741.

2. Il s'agit là d'une domination issue du regard lui-même, de l'acte d'observation simultané suscitant une hiérarchisation horizontale, depuis la salle vers la scène. Mais cette relation sera au cours de l'histoire plusieurs fois renversée, en fonction de l'évolution des conventions et des contraintes. Il est à noter par ailleurs que cette hiérarchisation se faisait à l'origine également dans la verticalité du fait que tous les actes ayant lieu au théâtre, lui-même prolongement du lieu sacrificiel, étaient considérés comme des gestes rituels soumis au regard de forces supérieures.

3. Dans un article pour la revue Marges, Laure Fernandez cite les sources suivantes pour situer la naissance du concept : «Ainsi Le Robert [...] date désormais sa première acception en 1842 ». Elle poursuit ainsi : «En 1908, le Russe Nicolas Evreinoff est l'un des premiers praticiens à consacrer une réflexion spécifique à la notion dans son essai Apologie de la théâtralité.", dans Fernandez Laure, «Théatralité et arts visuels : le paradoxe du spectateur. Autour de "The World as a Stage" et "Un teatre sense teatre" ", Marges [En ligne], 10|2010, mis en ligne le 15 avril 2010, URL: http://journals.openedition.org/marges/490; DOI : 10.4000/marges.490 [consulté le 23/05/2019].

4. Josette Féral, «La théâtralité: la spécificité du langage théâtral », Poétique, $\mathrm{n}^{\circ} 75$, Paris, Le Seuil, 1988, p. 347.

5. Patrice Pavis, "Théâtralité en Avignon ", Vers une théorie de la pratique théâtrale: voix et images de la scène, Paris, Presses Universitaires du Septentrion, 2000, p. 267.

6. Robert Morris (né en 1931), est un artiste plasticien et écrivain américain considéré comme l'un des fondateurs du mouvement minimaliste, ainsi que l'un de ses principaux théoriciens.

7. Michael Fried, "Art et objectité », Contre la théâtralité. Du minimalisme à la photographie contemporaine, Paris, Gallimard, 2007.

8. Patrice Pavis, "Théâtralité », Dictionnaire du théâtre, Paris, Armand Colin, 2004 ; et du même auteur, "Théâtralité en Avignon », op. cit.

9. Josette Féral, « La théâtralité : la spécificité du langage théâtral », op. cit, p. 347.

10. Ibid., p. 350.

11. Id.

12. Roland Barthes, Essais critiques, Paris, Le Seuil, 1964, p. 45.

13. Id.

14. Il s'agit en réalité plutôt de la réinterprétation de l'une de ses œuvres précédentes, Nightsea Crossing (1981), réalisée en collaboration avec Ulay, son collaborateur artistique pour 71 œuvres durant la période 1976-1988. Pour cette performance, les deux artistes étaient assis, immobiles, dans différents lieux, $9 \mathrm{~h}$ par jour pendant 90 jours.

15. Voir Christian Biet et Christophe Triau, «La mise en scène : un héritage, des interrogations », Qu'est-ce que le théâtre?, Paris, Gallimard, 2006.

16. Cette table sera retirée par la suite, l'artiste désirant réduire la distance qui la sépare du participant.

17. Georges Didi-Huberman, Peuples exposés, peuples figurants. L'œil de l'histoire, 4, Paris, Les Éditions de Minuit, 2012, p. 149.

18. Brian O'Doherty, White Cube. L'espace de la galerie et son idéologie, Zurich, JRP Ringier, 2008.

19. Ces relations et la perception qui en découle font de cette performance une œuvre qui permet, comme le dit Laure Fernandez, « de repenser de façon critique la notion de public et de communauté ». Cela est rendu possible par une implication du spectateur, non pas sous la forme d'une «participation physique (au sens de mouvante, actante), mais [d'une] réincarnation de l'œil », Fernandez Laure, op. cit., p. 35-36.

20. Gilles Girard, Ouellet Réal et Claude Rigault, L'Univers du Théâtre, Paris, Puf, 1978, p. 119.

21. Démosthènes Davvetas, philosophe de l'art ayant consacré un ouvrage à cette performance, décrit quelques réactions et comportements des participants vis-à-vis de ce regard passif : "Sans manifester le moindre affect mais le regard concentré, elle parvenait à provoquer une réaction 
chez chacun de ceux qui prenaient place face à elle. Certains étaient émus, d'autres demeuraient impassibles, la plupart fondaient en larmes, révélant publiquement et sans honte leur sensibilité, se perdant dans son regard, tandis que d'autres encore cherchaient à la provoquer, comme l'un d'eux qui s'était affublé d'un masque monstrueux ou cette jeune fille qui s'est déshabillée complètement et a voulu s'asseoir nue devant l'artiste.", Démosthènes Davvetas, Marina Abramović : méditation aux yeux fixes, Paris, Éditions Nicolas Chaudun, 2013, p. 78.

22. Cela fait notamment écho à une œuvre précédente de Marina Abramović, Rhythm 0 (1974), dans laquelle elle réalise une expérience sur le comportement dominant et le conditionnement du public, en exposant son corps et en le mettant à disposition face à soixante-douze objets plus ou moins dangereux manipulables par les visiteurs.

23. Voir Matthew Akers et Jeff Dupre, Marina Abramović, The Artist Is Present, film documentaire, New York, HBO Documentary Films, 2012, DVD.

24. Pour plus de détails à ce sujet, voir Anne Bénichou, «Marina Abramović : The Artist Is [Tele] Present : les nouveaux horizons photographiques de la (re)performance », Intermédialités, Québec, $n^{\circ}$ 17, 2011, p. 158. [En ligne] https://doi.org/10.7202/1005754ar [Consulté le 20/02/2019].

25. The Museum of Modern Art, Marina Abramović: The Artist Is Present-Portraits, Flickr, MoMA. [En ligne] : https://www.flickr.com/photos/themuseumofmodernart/sets/72157623741486824/ [Consulté le 30/08/2018].

26. Voir à ce propos l'entretien filmé avec Marina Abramović et Marco Anelli autour de l'ouvrage de Portraits in the Presence of Marina Abramović ayant eu lieu à la librairie Strand Bookstore à New York. [En ligne] https://www.youtube.com/watch?v=JdSs6KEH1OA [Consulté le 23/03/2019].

27. Rappelant la condition similaire du spectateur-participant face à l'artiste qui ne transmet aucune émotion.

28. Jay David Bolter et Richard Grusin, «Immediacy, Hypermediacy, and Remediation ", Remediation: Understanding new media, Cambridge, MIT Press, 2000.

29. Le concept de remédiation distingue deux voies: l'immédiateté (qui prône et produit la transparence du média) - et l'hypermédiateté (qui, au contraire, focalise l'attention sur les effets de multi-médiation). C'est de la première notion dont il sera question dans cette partie lorsque la remédiation sera évoquée.

30. Dans l'entretien autour de l'ouvrage de Portraits in the Presence of Marina Abramović évoqué plus haut, Marco Anelli expliquait son intention de diriger son travail vers un rendu plus « émotionnel » que « documentaire ».

31. Gilles Declercq, "Pathos et théâtralité. Pour une économie cognitive des passions ", in Michael Rinn (dir.), Émotions et discours: L'usage des passions dans la langue, [en ligne]. Rennes, Presses universitaires de Rennes, 2008 (généré le 23 mai 2019). Disponible sur Internet : <http:// books.openedition.org/pur/30443>. ISBN : 9782753546752. DOI : 10.4000/books.pur.30443 [consulté le 23/05/2019].

32. Id.

33. Cette performance peut en effet, et pour plusieurs raisons, être considérée comme synthétique de la rétrospective dans son ensemble. En premier lieu, le concept même de cette œuvre symbolise la présence de l'artiste elle-même, offrant au regard du public le corps témoin des expérimentations et transgressions présentées dans l'exposition. Aussi, elle exprime plusieurs thèmes récurrents des œuvres de l'artiste comme entre autres le comportement des spectateurs face à la performance ou la résistance du corps à l'épreuve du temps. 


\section{RÉSUMÉS}

La performance The Artist Is Present, créée par Marina Abramović en 2010, servira de point de départ à cet article pour tenter de démontrer comment la théâtralité influe sur la condition du spectateur et sa réception de l'œuvre. Cette théâtralité prendra ici des formes multiples et s'exprimera à plusieurs niveaux, instaurant des relations spectateur-œuvre complexes. La figure du spectateur y est en effet diverse, celui-ci étant à la fois observateur, participant et ultime récepteur d'une démarche artistique visant à le placer au centre de l'attention, à en faire à la fois le sujet et l'objet des regards suscités par la performance. Ainsi positionné, c'est finalement vers lui-même que sera retourné son regard, que ce soit par l'intermédiaire de celui de l'artiste ou en se projetant dans celui des participants qui lui est transmis par un système de diffusion numérique.

\section{INDEX}

Mots-clés : Marina Abramović, théâtralité, performance, spectateur, participation, regard, perception, remédiation, réception, phénomène perceptif, retournement

\section{AUTEUR}

\section{SUNGA KIM}

Doctorante en Esthétique, Sciences et Technologies des Arts à l'université Paris 8 , sa thèse porte sur Le retournement du regard dans l'art théâtral : les spectateurs regardés, sous la direction de $\mathrm{M}^{\mathrm{me}}$ Isabelle Moindrot. Elle a notamment été publiée dans l'ouvrage collectif L'Acteur face aux écrans, Corps en scène dirigé par Josette Féral (Paris, L'Entretemps, 2018) ; sunga.kim09@etud.univparis8.fr 